\title{
Revisit on two-dimensional self-gravitating kinks: superpotential formalism and linear stability
}

\author{
Yuan Zhong ${ }^{1}$ \\ School of Physics, Xi'an Jiaotong University, \\ Xi'an 710049, People's Republic of China \\ E-mail: zhongy@mail.xjtu.edu.cn
}

ABSTRACT: Self-gravitating kink solutions of a two-dimensional dilaton gravity are revisited in this work. Analytical kink solutions are derived from a concise superpotential formalism of the dynamical equations. A general analysis on the linear stability is conducted for an arbitrary static solution of the model. After gauge fixing, a Schrödinger-like equation with factorizable Hamiltonian operator is obtained, which ensures the linear stability of the solution.

KEYwords: 2D Gravity, Solitons Monopoles and Instantons

ArXiv EPrint: 2101.10928

\footnotetext{
${ }^{1}$ Corresponding author.
} 


\section{Contents}

1 Introduction 1

2 The model and the superpotential formalism 2

3 Exact solutions $\quad 4$

3.1 Reproducing Stötzel's solution 4

$\begin{array}{ll}3.2 \text { A polynomial superpotential } & 6\end{array}$

$\begin{array}{lll}4 & \text { Linear stability analysis } & 6\end{array}$

5 Summary and outlook 11

\section{Introduction}

During the past decades, two-dimensional (2D) gravitational models continue attracting the attention of theorists for a variety of reasons. First of all, the field equations obtained in many 2D gravity models are simple enough to allow a rigorous analysis of some difficult issues of gravitational theory, such as the quantization of gravity [1, 2], gravitational collapse $[3,4]$, black hole evaporation [5-9], see [10-12] for comprehensive reviews on early works. Second, a number of very different approaches of quantum gravity all hint that at very short distances space-time becomes effectively two dimensional [13-18]. Here, the dimensions that are reduced can be effective, spectral, topological or the usual dimensions [19]. Recently, the studies of the Sachdev-Ye-Kitaev (SYK) model [20-22] also lead to a resurgence of interest in 2D gravity [23-26], see [27-29] for pedagogical introductions.

Since the Einstein tensor vanishes identically in two dimensions, the Einstein-Hilbert action cannot be used to describe $2 \mathrm{D}$ gravity. An economical solution to this problem is to introduce a dilaton field. Many different 2D dilaton gravity models have been proposed and studied so far. The simplest action for 2D dilaton gravity is the Jackiw-Teitelboim (JT) action $[30,31]$

$$
S_{\mathrm{JT}}=\frac{1}{\kappa} \int d^{2} x \sqrt{-g} \varphi(R+\Lambda),
$$

where the dilaton $\varphi$ plays the role of a Lagrangian multiplier. $\kappa$ and $\Lambda$ are the gravitational coupling and the cosmological constant, respectively. Two other famous actions for 2D dilaton gravity are the Mann-Morsink-Sikkema-Steele (MMSS) action, which generalize the JT action by giving the dilaton a kinetic term [32]

$$
S_{\mathrm{MMSS}}=\frac{1}{\kappa} \int d^{2} x \sqrt{-g}\left[-\frac{1}{2}(\nabla \varphi)^{2}+\varphi R+\Lambda\right],
$$


and the Callan-Giddings-Harvey-Strominger (CGHS) action [5]:

$$
S_{\mathrm{CGHS}}=\frac{1}{2 \pi} \int d^{2} x \sqrt{-g}\left\{e^{-2 \varphi}\left[R+4(\nabla \varphi)^{2}+4 \Lambda^{2}\right]-\frac{1}{2}(\nabla \phi)^{2}\right\}
$$

where $\phi$ is a massless scalar matter field. A comprehensive review of $2 \mathrm{D}$ dilaton gravity models and their applications in black hole physics and quantum gravity can be found in ref. [12].

It is a natural idea to extend the discussion on 2D dilaton gravity to other classical solutions such as topological solitons, which could be produced by cosmic phase transitions [33]. As the simplest topological soliton solution, kink (or domain wall) has been extensively studied in 4D cosmology [34] and 5D thick brane world models [35, 36]. In the case of two dimensions, previous works have revealed close connections between kinks and 2D black holes [37-40], or naked singularities [3, 4, 41-43].

In 1995, an exact 2D self-gravitating sine-Gordon kink solution without curvature singularity was found by Stötzel, in the MMSS gravity model [44]. In addition to the kink configuration of the scalar field, the metric solution [44] describes a 2D asymptotic anti de-Sitter $\left(\mathrm{AdS}_{2}\right)$ geometry. This property reminds us the thick brane solutions found in asymptotic $\mathrm{AdS}_{5}$ geometry [45-47]. The aim of the present work is to reveal similarities between 2D self-gravitating kinks and 5D thick brane worlds.

The organization of the paper is as follows. In section 2 , we give a brief review of Stötzel's model, and show that for static solutions, the field equations can be written as a group of first-order differential equations by introducing the so called superpotential. With the superpotential formalism, one can easily generate exact self-gravitating kink solutions by chosen proper superpotentials. We will discuss two analytical solutions in section 3 . Then, in section 4 we give a complete analysis to the linear stability of the solutions. To our knowledge, no such analysis was done before. In a recent work [48], the authors considered the linear perturbations around self-gravitating kink solutions in 2D MMSS gravity. However, they expand the metric around the Minkowski metric rather than the asymptotic $\mathrm{AdS}_{2}$ metric solution. Finally, we offer in section 5 some concluding remarks.

\section{The model and the superpotential formalism}

The action of Stötzel's model [44] contains an MMSS gravity part along with a canonical real scalar $\phi$ :

$$
S=\frac{1}{\kappa} \int d^{2} x \sqrt{-g}\left[-\frac{1}{2} \partial^{\mu} \varphi \partial_{\mu} \varphi+\varphi R+\Lambda+\kappa \mathcal{L}_{\mathrm{m}}\right]
$$

where

$$
\mathcal{L}_{\mathrm{m}}=-\frac{1}{2} \partial^{\mu} \phi \partial_{\mu} \phi-V(\phi)
$$

is the Lagrangian density of the scalar field.

After variation, one immediately obtains the Einstein equations

$$
\nabla_{\mu} \varphi \nabla_{\nu} \varphi+2 \nabla_{\mu} \nabla_{\nu} \varphi-\frac{1}{2} g_{\mu \nu}\left(\nabla_{\lambda} \varphi \nabla^{\lambda} \varphi+4 \nabla_{\lambda} \nabla^{\lambda} \varphi-2 \Lambda\right)=-\kappa T_{\mu \nu}
$$


the dilaton equation

$$
\nabla_{\lambda} \nabla^{\lambda} \varphi+R=0
$$

and the scalar field equation

$$
\nabla^{\mu} \nabla_{\mu} \phi-\frac{d V}{d \phi}=0
$$

The energy-momentum tensor in eq. (2.3) is defined as

$$
\begin{aligned}
T_{\mu \nu} & =g_{\mu \nu} \mathcal{L}_{\mathrm{m}}-2 \frac{\delta \mathcal{L}_{\mathrm{m}}}{\delta g^{\mu \nu}} \\
& =\partial_{\mu} \phi \partial_{\nu} \phi-\frac{1}{2} g_{\mu \nu}\left(\partial^{\alpha} \phi \partial_{\alpha} \phi+2 V\right) .
\end{aligned}
$$

To obtain self-gravitating kink solution, Stötzel used the following metric

$$
d s^{2}=-e^{2 A(x)} d t^{2}+d x^{2} .
$$

Similar metric ansatz is also used in 5D brane world models with non-factorizable geometry $[49,50]$, therefore, we will follow the terminology of brane world theory and call the function $A(x)$ as the warp factor. As a convention, the derivative with respect to $x$ will always be denoted as a subscript, for example, $\phi_{x} \equiv d \phi / d x$.

Substituting metric (2.7) into the Einstein equations (2.3), one obtains

$$
\begin{aligned}
2 A_{x} \varphi_{x}-2 \varphi_{x x}-\varphi_{x}^{2} & =\kappa \phi_{x}^{2}, \\
A_{x} \varphi_{x}+\varphi_{x x} & =\Lambda-\kappa V .
\end{aligned}
$$

The equations of motion for the dilaton and the scalar fields read

$$
-2 A_{x x}-2 A_{x}^{2}+\varphi_{x x}+A_{x} \varphi_{x}=0 .
$$

and

$$
A_{x} \phi_{x}+\phi_{x x}=\frac{d V}{d \phi}
$$

respectively. Note that only three of the above equations are independent. For example, eq. (2.11) can be derived by using eqs. (2.8)-(2.10). At a first glance, eqs. (2.8)-(2.11) constitute a complicate nonlinear differential system, and finding their solutions seems to be a formidable task. But the study of brane world models has taught us a lesson on how to solve such system by means of superpotential method, which rewrites second-order differential equations, such as eqs. (2.8)-(2.11), into some first-order ones [45-47].

To construct a superpotential formalism for the present model, we first note that the combination of eqs. (2.9) and (2.10) leads to an expression of $V$ in terms of cosmological constant and warp factor:

$$
\kappa V=\Lambda-2 A_{x x}-2 A_{x}^{2}
$$

Taking the derivative of the above equation and eliminating $d V / d \phi$ by using eq. (2.11), one obtains a relation between $A$ and $\phi$ :

$$
A_{x x x}+2 A_{x} A_{x x}=-\frac{1}{2} \kappa\left(A_{x} \phi_{x}^{2}+\phi_{x x} \phi_{x}\right) .
$$


The superpotential method starts with an assumption that the first-order derivative of $\phi$ equals to a function of $\phi$ itself, namely, the superpotential $W(\phi)$ via the following equation:

$$
\phi_{x}=\frac{d W}{d \phi} .
$$

Under this assumption, one can testify that eq. (2.13) supports a very simple special solution:

$$
A_{x}=-\frac{1}{4} \kappa W
$$

Then, eq. (2.12) enables us to write $V$ in terms of superpotential:

$$
V=\frac{1}{2}\left(\frac{d W}{d \phi}\right)^{2}-\frac{1}{8} \kappa W^{2}+\frac{\Lambda}{\kappa} .
$$

Finally, the general solution of eq. (2.10) gives a simple relation between dilaton and warp factor:

$$
\varphi=2 A+\beta \int e^{-A} d x+\varphi_{0},
$$

where $\beta$ and $\varphi_{0}$ are just two integral constants. Since the field equations only contain the derivatives of the dilaton, the value of $\varphi_{0}$ is unimportant to the solution of other variables, and can be taken as $\varphi_{0}=0$. Besides, to consist with eq. (2.8), $\beta$ must be set as zero, so

$$
\varphi=2 A
$$

Eqs. (2.14)-(2.17) constitute the first-order superpotential formalism of the present model. Exact kink solutions can be derived by choosing proper superpotentials. The freedom of choosing a superpotential comes from the fact that there are four unknown variables $(A, \phi, \varphi$ and $V)$ but only three independent equations. Taking a superpotential amounts to specifying one of the four unknown variables.

\section{Exact solutions}

In this section, we show how to use the superpotential formalism to derive exact selfgravitating kink solutions. We first reproduce Stötzel's solution and then report a new solution.

\subsection{Reproducing Stötzel's solution}

In fact, the superpotential formalism presented in last section has been derived and used, although unconsciously, by Stötzel [44]. Instead of choosing a superpotential $W(\phi)$, Stötzel started with the Sine-Gordon potential

$$
V(\phi)=2 m^{2} \sin ^{2} \frac{\phi}{2} .
$$

He observed that when $\kappa=\frac{\lambda}{4 m^{2}-\lambda}$, eq. (2.16) surports two solutions of the superpotential:

$$
W_{ \pm}= \pm 2 \sqrt{4 m^{2}-\lambda} \cos \left(\frac{\phi}{2}\right)
$$


(a)

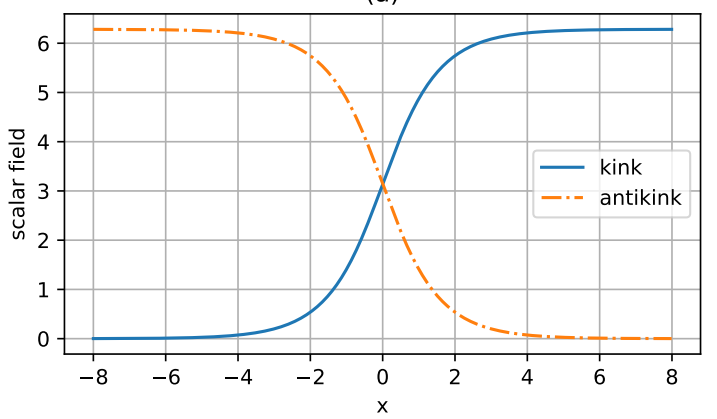

(b)

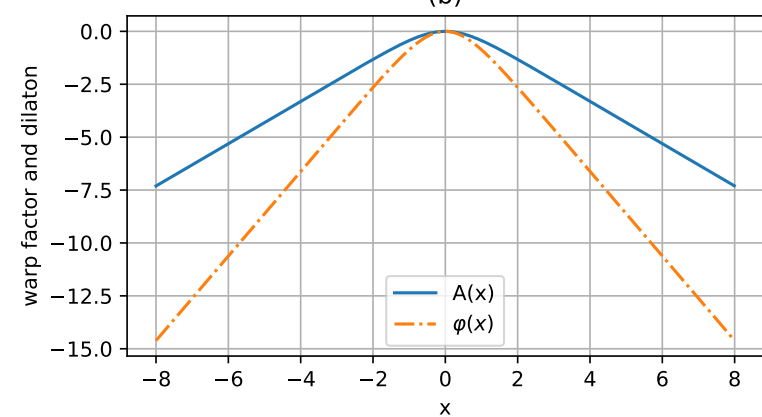

Figure 1. The shapes of some important variables in Stötzel's solution, incluting (a) scalar field, (b) warp factor and the dilaton field. The parameters are taken as $\kappa=1, m=\sqrt{2}$ and $\lambda=4$, therefore $M=1$ and $\Lambda=2$.

where $0<\lambda \equiv \frac{2 \Lambda}{\kappa}<4 m^{2}$. The solutions of $\phi(x)$ corresponds to $W_{-}$could be obtained by integrating eqs. (2.14), and the result turns out to be the sine-Gordon kink [44]:

$$
\phi_{K}(x)=4 \arctan \left(e^{M\left(x-x_{0}\right)}\right) .
$$

Here $x_{0}$ is an integral constant that represents the position of the kink, and will be set to zero from now on. The constant $M$ is defined as $M \equiv \frac{1}{2} \sqrt{4 m^{2}-\lambda}$. Obviously, $M \in(0, m)$. The solution corresponds to $W_{+}$is an antikink

$$
\phi_{\bar{K}}(x)=4 \arctan \left(e^{-M x}\right),
$$

which is similar as the kink in many aspects. Thus, we will focus on the kink solution only, and eliminate the subscript $K$ from now on.

Plugging the solutions of $W(\phi)$ and $\phi(x)$ into eq. (2.15), one immediately obtains the expression of the warp factor:

$$
A(x)=A_{0}-\frac{\lambda}{4 M^{2}} \ln (2 \cosh (M x)),
$$

which further reduces to [44]

$$
\begin{aligned}
A(x) & =-\frac{\lambda}{4 M^{2}} \ln \cosh (M x) \\
& =-\kappa \ln \cosh (M x)
\end{aligned}
$$

after taking integral constant $A_{0}=\frac{\lambda}{4 M^{2}} \ln 2$. Obviously, this warp factor describes an asymptotic $\mathrm{AdS}_{2}$ geometry. Finally, the dilaton field reads

$$
\varphi(x)=2 A(x)=-2 \kappa \ln \cosh (M x) .
$$

The profiles of $\phi, A$ and $\varphi$ are plotted in figure 1. 


\subsection{A polynomial superpotential}

As shown repeatedly in the study of $5 \mathrm{D}$ thick brane models, it is quite easy to construct exact self-gravitating kink solutions once the superpotential formalism is established. In the following discussions, we will take $\Lambda=0$ for simplicity, as it can be absorbed into the definition of $V(\phi)$.

Consider a simple polynomial potential with parameter $c$ [51-53]

$$
W=c+\phi\left(1-\frac{\phi^{2}}{3}\right) .
$$

It has two minima at $\phi_{ \pm}= \pm 1$, where $W\left(\phi_{ \pm}\right)= \pm \frac{2}{3}+c$. With this superpotential, one obtains [53]

$$
\begin{aligned}
\phi(x) & =\tanh (x) \\
\varphi(x) & =2 A(x) \\
A(x) & =\frac{1}{24} \kappa\left[-6 c x+\operatorname{sech}^{2}(x)-4 \ln (\cosh (x))-1\right], \\
V(\phi) & =-\frac{1}{72} \kappa\left(-3 c+\phi^{3}-3 \phi\right)^{2}+\frac{1}{2}\left(\phi^{2}-1\right)^{2} .
\end{aligned}
$$

The asymptotic behaviors of the warp factor and the scalar potential are

$$
\begin{aligned}
A_{ \pm}(x) & =-\frac{1}{4} \kappa W\left(\phi_{ \pm}\right) x=-\frac{1}{4} \kappa\left(\frac{2}{3} \pm c\right)|x|, \\
V_{ \pm} & =-\frac{1}{72}(3 c \pm 2)^{2} \kappa .
\end{aligned}
$$

Depending on the value of $c$, there are four different situations [53]:

1. $c=0$ : in this case, the kink connects two equivalent $\mathrm{AdS}_{2}$ spaces symmetrically, and $V_{+}=V_{-}=-\frac{1}{18} \kappa$.

2. $0<|c|<\frac{2}{3}$ : the kink connects two distinct $\mathrm{AdS}_{2}$ spaces.

3. $|c|=\frac{2}{3}$ : the kink connects an $\mathrm{AdS}_{2}$ space and a 2D Minkowski space $\left(\mathrm{M}_{2}\right)$ asymmetrically. This situation is of particular interesting when considering kink collision in asymptotical AdS space-time $[52,54]$.

4. $|c|>\frac{2}{3}$ : the warp factor diverges at one side of the kink.

The behavior of $e^{A}$ for different values of $c$ has been plotted in figure 2. Obviously, for $c \neq 0$, the warp factor is asymmetric.

\section{Linear stability analysis}

In this section, we discuss the linear stability of the self-gravitating kink solutions against small perturbations. This issue has been studied extensively in 5D brane world models $[46,55-58]$, but remains untouched in the case of 2D. The reducing of dimensions and 


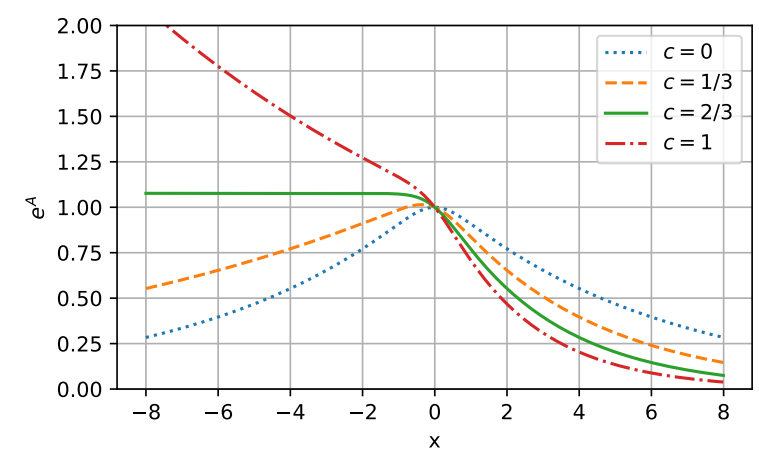

Figure 2. Plots of warp factor $e^{A(x)}$ of the polynomial model with $\kappa=1$.

the introducing of dilaton field make it impossible to analyze linear stability of $2 \mathrm{D}$ selfgravitating kinks by simply copying the stability analysis of 5D thick branes. For example, there are no vector and tensor perturbation in $2 \mathrm{D}$, so the traditional scalar-vector-tensor decomposition $[56,58]$ is no longer needed. Beside, in 2D there is no way to eliminate the non-minimal gravity-dilaton coupling by using conformal transformation.

It is convenient to discuss the linear stability in the conformal flat coordinates

$$
d s^{2}=e^{2 A(r)} \eta_{\mu \nu} d x^{\mu} d x^{\nu}
$$

where $r$ is defined through $d r \equiv e^{-A(x)} d x$. For simplicity, we use a prime and an overdot to represent the derivatives with respect to $r$ and $t$, respectively.

In this coordinates, the Einstein equations take the following form:

$$
\begin{aligned}
\kappa \phi^{\prime 2} & =4 A^{\prime} \varphi^{\prime}-2 \varphi^{\prime \prime}-\varphi^{\prime 2}, \\
\varphi^{\prime \prime} & =e^{2 A}(\Lambda-\kappa V) .
\end{aligned}
$$

The equation of motion for the scalar and dilaton fields are

$$
\phi^{\prime \prime}=e^{2 A} \frac{d V}{d \phi},
$$

and

$$
\varphi^{\prime \prime}=2 A^{\prime \prime}
$$

respectively. Obviously, the general solution of eq. (4.5) is $\varphi=2 A+\beta r+\varphi_{0}$, but as stated before, we will take $\beta=0=\varphi_{0}$.

Equation (2.13) becomes

$$
2 A^{\prime \prime \prime}-4 A^{\prime} A^{\prime \prime}+\kappa \phi^{\prime} \phi^{\prime \prime}=0
$$

which, after integration, gives

$$
A^{\prime \prime}-A^{\prime 2}+\frac{1}{4} \kappa \phi^{\prime 2}=0,
$$

where the integral constant has been taken as zero. 
Now, let us consider small field perturbations around an arbitrary static background solution $\left\{\varphi(r), \phi(r), g_{\mu \nu}(r)\right\}$ :

$$
\varphi(r)+\delta \varphi(r, t), \quad \phi(r)+\delta \phi(r, t), \quad g_{\mu \nu}(r)+\delta g_{\mu \nu}(r, t) .
$$

We also define

$$
\delta g_{\mu \nu}(r, t) \equiv e^{2 A(r)} h_{\mu \nu}(r, t)
$$

for convenience.

In the linear perturbation analysis of cosmological or brane world models, one usually decompose $h_{\mu \nu}$ into scalar, vector and tensor sectors [59, 60]. Each sector can be discussed independently. In the present case, we have only one spatial dimension and no such decomposition is needed. So we will directly deal with the components of the metric perturbation

$$
h_{\mu \nu}=\left(\begin{array}{cc}
h_{00}(r, t) & \Phi(r, t) \\
\Phi(r, t) & h_{r r}(r, t)
\end{array}\right),
$$

where we have renamed $h_{01}=h_{10}$ as $\Phi$, and $h_{11}$ as $h_{r r}$.

To the first order, the perturbation of the metric inverse is given by

$$
\delta g^{\mu \nu}=-e^{-2 A} h^{\mu \nu} .
$$

Note that the indices of $h$ are always raised or lowered with $\eta_{\mu \nu}$, thus,

$$
h^{\mu \nu} \equiv \eta^{\mu \rho} \eta^{\nu \sigma} h_{\rho \sigma}=\left(\begin{array}{cc}
h_{00} & -\Phi \\
-\Phi & h_{r r}
\end{array}\right) .
$$

After linearization, the Einstein equations (2.3) lead to three nontrivial perturbation equations, namely, the $(0,0)$ component:

$$
\begin{aligned}
& 2 A^{\prime} \delta \varphi^{\prime}-2 A^{\prime} \varphi^{\prime} h_{r r}-2 \delta \varphi^{\prime \prime}-\delta \varphi^{\prime} \varphi^{\prime}+h_{r r}^{\prime} \varphi^{\prime} \\
& +2 h_{r r} \varphi^{\prime \prime}+\frac{1}{2} h_{r r} \varphi^{\prime 2}=\kappa\left(\phi^{\prime} \delta \phi^{\prime}+\phi^{\prime \prime} \delta \phi-\frac{1}{2} \phi^{2} h_{r r}\right),
\end{aligned}
$$

the $(0,1)$ or $(1,0)$ components:

$$
2 A^{\prime} \delta \varphi-2 \delta \varphi^{\prime}-\varphi^{\prime} \delta \varphi+\varphi^{\prime} h_{r r}=\kappa \phi^{\prime} \delta \phi,
$$

and the $(1,1)$ component:

$$
2 A^{\prime} \delta \varphi^{\prime}-2 A^{\prime} \varphi^{\prime} h_{r r}-\delta \varphi^{\prime} \varphi^{\prime}-2 \ddot{\delta} \varphi+\frac{1}{2} h_{r r} \varphi^{\prime 2}+\Xi \varphi^{\prime}=\kappa\left(\phi^{\prime} \delta \phi^{\prime}-\phi^{\prime \prime} \delta \phi-\frac{1}{2} \phi^{2} h_{r r}\right) .
$$

Here we have defined a new variable $\Xi \equiv 2 \dot{\Phi}-h_{00}^{\prime}$. One can testify that after using background equations (4.2)-(4.5), eq. (4.13) reduces to eq. (4.14).

Another independent equation comes from the perturbation of the scalar equation of motion:

$$
-\ddot{\delta} \phi+\delta \phi^{\prime \prime}+2 A^{\prime} \frac{\phi^{\prime \prime}}{\phi^{\prime}} \delta \phi-\frac{\phi^{\prime \prime \prime}}{\phi^{\prime}} \delta \phi-\frac{1}{2} \phi^{\prime} h_{r r}^{\prime}-\phi^{\prime \prime} h_{r r}+\frac{1}{2} \phi^{\prime} \Xi=0 .
$$

One can also linearize the dilaton equation (2.4), but it does not offer new information further. 
Therefore, we have three independent perturbation equations, i.e., (4.14)-(4.16). But one should note that the perturbation variables are not all independent. The invariance of the dynamical equations under coordinate transformations

$$
x^{\mu} \rightarrow \tilde{x}^{\mu}=x^{\mu}+\xi^{\mu}(r, t)
$$

induces an invariance of the linear perturbation equations (4.14)-(4.16) under the following gauge transformations:

$$
\begin{aligned}
\Delta h_{\mu \nu} & \equiv \widetilde{h}_{\mu \nu}-h_{\mu \nu}=-2 \xi_{(\mu, \nu)}-2 \eta_{\mu, \nu} A^{\prime} \xi^{1} \\
\Delta \delta \phi & \equiv \widetilde{\delta \phi}-\delta \phi=-\phi^{\prime} \xi^{1} \\
\Delta \delta \varphi & \equiv \widetilde{\delta \varphi}-\delta \varphi=-\varphi^{\prime} \xi^{1} .
\end{aligned}
$$

The components of $h_{\mu \nu}$ transform as

$$
\begin{aligned}
\Delta h_{00} & =2 \partial_{t} \xi^{0}+2 A^{\prime} \xi^{1}, \\
\Delta \Phi & =-\partial_{t} \xi^{1}+\partial_{r} \xi^{0}, \\
\Delta h_{r r} & =-2 \partial_{r} \xi^{1}-2 A^{\prime} \xi^{1},
\end{aligned}
$$

which means that the variable $\Xi=2 \dot{\Phi}-h_{00}^{\prime}$ should transforms as

$$
\Delta \Xi=-2\left[\ddot{\xi}^{1}+\left(A^{\prime} \xi^{1}\right)^{\prime}\right] .
$$

We see that the gauge degree of freedom $\xi^{0}$ has been canceled.

The residual gauge degree of freedom in $\xi^{1}$ allows us to eliminate one of the perturbation variables. Here we simply take $\delta \varphi=0$, with which eq. (4.14) reduces to

$$
\varphi^{\prime} h_{r r}=\kappa \phi^{\prime} \delta \phi
$$

and eq. (4.15) becomes

$$
-2 A^{\prime} \varphi^{\prime} h_{r r}+\frac{1}{2} h_{r r} \varphi^{\prime 2}+\Xi \varphi^{\prime}=\kappa\left(\phi^{\prime} \delta \phi^{\prime}-\phi^{\prime \prime} \delta \phi-\frac{1}{2} \phi^{\prime 2} h_{r r}\right) .
$$

After eliminating $h_{r r}$ and $\Xi$, equation (4.16) can be written as a wave equation of $\delta \phi$ :

$$
\ddot{\delta \phi}-\delta \phi^{\prime \prime}+V_{\text {eff }}(r) \delta \phi=0,
$$

where the effective potential reads

$$
V_{\mathrm{eff}}(r)=4 A^{\prime \prime}-2 A^{\prime} \frac{\phi^{\prime \prime}}{\phi^{\prime}}-\varphi^{\prime \prime}+2\left(\frac{\varphi^{\prime \prime}}{\varphi^{\prime}}\right)^{2}-2 \frac{\varphi^{\prime \prime \prime}}{\varphi^{\prime}}+\frac{\phi^{\prime \prime \prime}}{\phi^{\prime}} .
$$

Using eqs. (4.5)-(4.7), one can obtain an useful identity:

$$
\varphi^{\prime \prime}=\frac{\varphi^{\prime \prime \prime}}{\varphi^{\prime}}+\frac{\phi^{\prime \prime}}{\phi^{\prime}} \varphi^{\prime}-2 \frac{\phi^{\prime \prime}}{\phi^{\prime}} \frac{\varphi^{\prime \prime}}{\varphi^{\prime}},
$$

which enable us to rewrite the effective potential as

$$
V_{\mathrm{eff}}=\frac{\phi^{\prime \prime \prime}}{\phi^{\prime}}-2 \frac{\phi^{\prime \prime}}{\phi^{\prime}} \frac{\varphi^{\prime \prime}}{\varphi^{\prime}}+2\left(\frac{\varphi^{\prime \prime}}{\varphi^{\prime}}\right)^{2}-\frac{\varphi^{\prime \prime \prime}}{\varphi^{\prime}},
$$

or, in a more compact form

$$
V_{\mathrm{eff}}=\frac{f^{\prime \prime}}{f}, \quad \text { with } \quad f \equiv \frac{\phi^{\prime}}{\varphi^{\prime}} .
$$


If we take $\delta \phi=\psi(r) e^{i w t}$, eq. (4.27) becomes a Schrödinger-like equation of $\psi(r)$ :

$$
-\psi^{\prime \prime}+V_{\text {eff }} \psi=w^{2} \psi
$$

It is interesting to note that the Hamiltonian operator are factorizable:

$$
\hat{H}=-\frac{d^{2}}{d r^{2}}+V_{\text {eff }}=\hat{\mathcal{A}} \hat{\mathcal{A}}^{\dagger},
$$

with

$$
\mathcal{A}=\frac{d}{d r}+\frac{f^{\prime}}{f}, \quad \mathcal{A}^{\dagger}=-\frac{d}{d r}+\frac{f^{\prime}}{f} .
$$

According to the theory of supersymmetric quantum mechanics [61], the eigenvalues of a factorizable Hamiltonian operator are semipositive definite, namely, $w^{2} \geq 0$. Therefore, static kink solutions are stable against linear perturbations. The zero mode $\left(w_{0}=0\right)$ satisfies $\mathcal{A}^{\dagger} \psi_{0}(r)=0$, and the solution reads

$$
\psi_{0}(r) \propto f=\frac{\phi^{\prime}}{\varphi^{\prime}}=\frac{\phi^{\prime}}{2 A^{\prime}} .
$$

Obviously, for any solution with a non-monotonic warp factor, $\psi_{0}(r)$ diverges at the extrema of $A$, and would be unnormalizable. Since it is not always possible to obtain the explicit expression of $x(r)$, it is useful to transform $V_{\text {eff }}$ back to the $x$-coordinates:

$$
V_{\text {eff }}(x)=e^{2 A}\left(A_{x} \frac{f_{x}}{f}+\frac{f_{x x}}{f}\right),
$$

with $f(x)=\phi_{x} / \varphi_{x}$.

It should be note that the stability analysis presented so far are rather general and does not depend on the specific form of the solution, but only on the general form of the metric (4.1) and of the action (2.1).

Now, we move on to the specific solutions. For Stötzel's sine-Gordon model and the polynomial model, the effective potentials read

$$
V_{\mathrm{eff}}(x)=M^{2} \cosh ^{-2 \kappa}(M x)\left[\kappa+2 \operatorname{csch}^{2}(M x)+1\right],
$$

and

$$
\begin{aligned}
V_{\mathrm{eff}}(x)= & \frac{\exp \left[\frac{1}{12}\left(-6 c x+\operatorname{sech}^{2}(x)-1\right)\right]}{12 \sqrt[3]{\cosh (x)}\left[3 c+\tanh (x)\left(\operatorname{sech}^{2}(x)+2\right)\right]^{2}}\left\{-\operatorname{sech}^{2}(x)[296\right. \\
& \left.+702 c^{2}+\left(27 c^{2}-424\right) \operatorname{sech}^{2}(x)+118 \operatorname{sech}^{4}(x)+\operatorname{sech}^{6}(x)+\operatorname{sech}^{8}(x)\right] \\
& \left.+18 c \tanh (x)\left[3 c^{2}+23 \operatorname{sech}^{4}(x)-32 \operatorname{sech}^{2}(x)+36\right]+540 c^{2}+208\right\}
\end{aligned}
$$

respectively. For the later case, we have taken $\kappa=1$, for simplicity.

The profiles of the $V_{\text {eff }}(x)$ are depicted in figure 3. For Stötzel's model, we take $m=\sqrt{2}$, $\Lambda=2 \kappa$ such that $M \equiv \frac{1}{2} \sqrt{4 m^{2}-\frac{2 \Lambda}{\kappa}}=1$, while keep $\kappa$ as a free parameter. We see that $V_{\text {eff }}$ is positive and divergent at $x=0$ for $\kappa=0.2,1$ and 3 . 

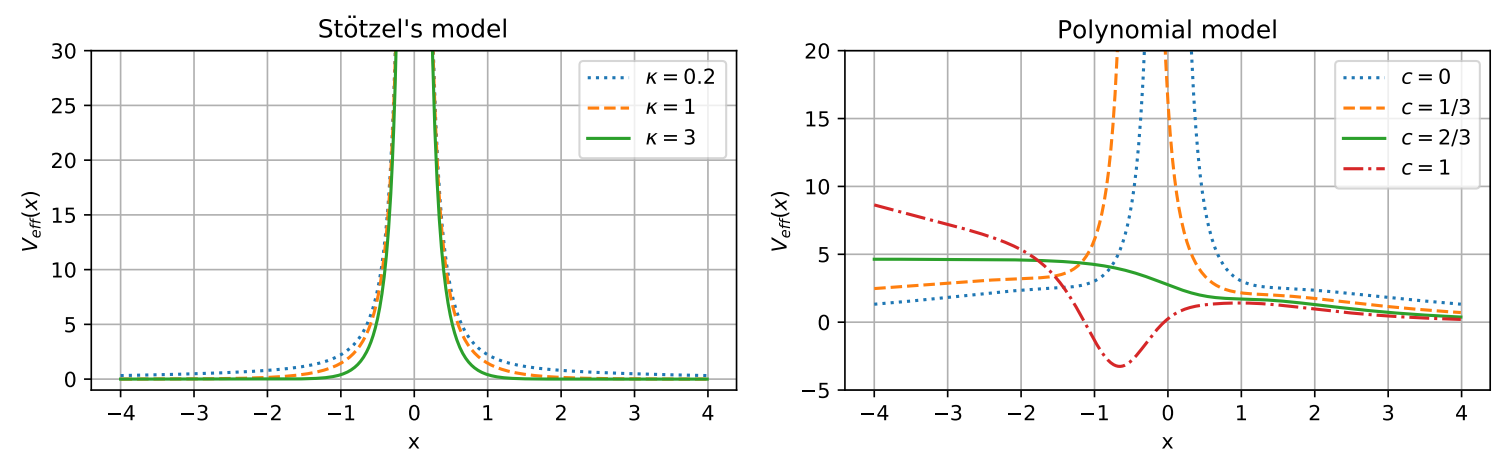

Figure 3. Plots of $V_{\text {eff }}(x)$. For polynomial model with $c=2 / 3, V_{\text {eff }}(x)$ becomes finite, and approaches to $4 \sqrt[3]{2} e^{-\frac{1}{12}} \approx 4.637$ as $x \rightarrow-\infty$.

For the polynomial model, we take $c=0,1 / 3,2 / 3$ and 1 as examples. We see that $V_{\text {eff }}(x)$ diverges at $x=0$ for both $c=0$ and $1 / 3$, while blows up at $x \rightarrow-\infty$ if $c=1$, but becomes finite when $c=2 / 3$.

It is worth to mention that in many $5 \mathrm{D}$ thick brane models the effective potentials of the scalar perturbation also have singularities, and the corresponding scalar zero modes are usually unnormalizable. Without normalizable scalar zero modes, these models are free of the problem of long range scalar fifth force [55, 56, 58]. For the 2D self-gravitating kink solutions considered in this paper, however, we find an unusual situation where the zero mode might be normalizable, namely, the polynomial model with $c>2 / 3$. In this case, the zero mode reads

$$
\psi_{0}(x)=\mathcal{N} \frac{\phi_{x}}{2 A_{x}}=-\mathcal{N} \frac{6 \operatorname{sech}^{2}(x)}{3 c+\tanh (x)\left(\operatorname{sech}^{2}(x)+2\right)},
$$

where $\mathcal{N}$ is the normalization constant, and we have taken $\kappa=1$. The normalization of zero mode requires

$$
1=\int_{-\infty}^{+\infty} d r \psi_{0}^{2}(r)=\mathcal{N}^{2} \int_{-\infty}^{+\infty} d x e^{-A}\left(\frac{\phi_{x}}{2 A_{x}}\right)^{2}
$$

The integration can be done numerically, for instance, taking $c=1,1.2$ and 1.5 we obtain $|\mathcal{N}| \approx 0.334,0.446$ and 0.598 , respectively. Plots of $\psi_{0}(x)$ is depicted in figure 4 .

\section{Summary and outlook}

In this work, we revisited smooth self-gravitating kink solutions of a type of 2D dilaton gravity proposed by Mann et al. [32]. We first showed that exact kink solutions can be constructed with the aid of a first-order superpotential formalism (2.14)-(2.17) of the dynamical equations. This formalism has already been derived and used by Stötzel in 1995, for 2D self-gravitating sine-Gordon model [44], but its virtue was not completely appreciated until the advent of 5D thick brane world models. After reproducing Stötzel's solution [44], we reported another kink solution generated by a polynomial superpotential used in some $5 \mathrm{D}$ brane world models [51-53]. 


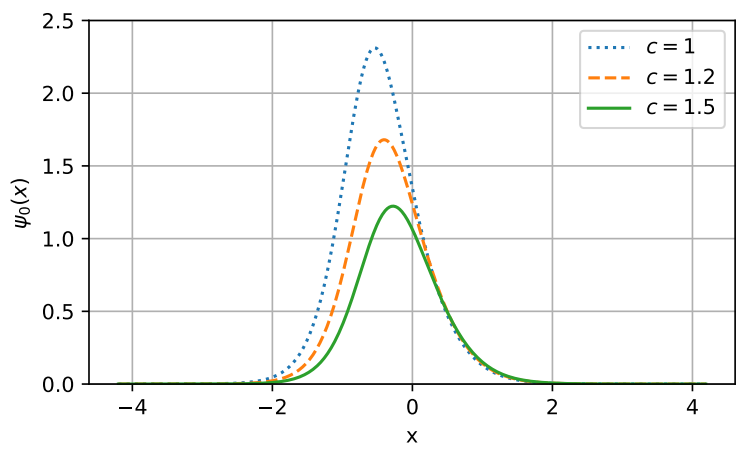

Figure 4. Plots of $\psi_{0}(x)$ for the polynomial model with $\kappa=1, c=1,1.2$ and 1.5.

The main contribution of the present work, however, is a general analysis on the stability of static kink solutions under small linear perturbations. After eliminating the redundant gauge degrees of freedom, we derived a Schrödinger-like equation for the physical perturbation. We found that the Hamiltonian operator can be factorized as $\hat{H}=\hat{\mathcal{A}} \hat{\mathcal{A}}^{\dagger}$, which implies the stability of the solutions. Besides, the zero mode takes the form $\psi_{0}(r) \propto f \equiv \frac{\phi^{\prime}}{\varphi^{\prime}}=\frac{\phi^{\prime}}{2 A^{\prime}}$, which diverges at the extrema of $A$. For Stötzel's model, the zero mode is not normalizable, because the symmetric solution of the warp factor corresponds to a singularity of $\psi_{0}(r)$ at $r=0$. For the polynomial model, however, the zero mode is normalizable provides $c>2 / 3$.

It is natural to ask if the superpotential formalism and stability analysis of the present work can also be extended to other 2D dilaton gravity models, such as the CGHS model [5] or other more general models $[62,63]$. The superpotential formalism also makes it possible discuss the application of the present model to the study of holographic RG flow [64, 65]. We will leave these questions to our future works.

\section{Acknowledgments}

This work was supported by the National Natural Science Foundation of China (Grant Nos. 11847211, 11605127), Fundamental Research Funds for the Central Universities (Grant No. xzy012019052), and China Postdoctoral Science Foundation (Grant No. 2016M592770).

Open Access. This article is distributed under the terms of the Creative Commons Attribution License (CC-BY 4.0), which permits any use, distribution and reproduction in any medium, provided the original author(s) and source are credited.

\section{References}

[1] M. Henneaux, Quantum Gravity in Two-Dimensions: Exact Solution of The Jackiw Model, Phys. Rev. Lett. 54 (1985) 959 [inSPIRE].

[2] S.P. de Alwis, Quantization of a theory of 2-D dilaton gravity, Phys. Lett. B 289 (1992) 278 [hep-th/9205069] [INSPIRE]. 
[3] C. Vaz and L. Witten, Formation and evaporation of a naked singularity in 2-D gravity, Phys. Lett. B 325 (1994) 27 [hep-th/9311133] [INSPIRE].

[4] C. Vaz and L. Witten, Do naked singularities form?, Class. Quant. Grav. 13 (1996) L59 [gr-qc/9511018] [INSPIRE].

[5] C.G. Callan Jr., S.B. Giddings, J.A. Harvey and A. Strominger, Evanescent black holes, Phys. Rev. D 45 (1992) R1005 [hep-th/9111056] [INSPIRE].

[6] A. Bilal and C.G. Callan Jr., Liouville models of black hole evaporation, Nucl. Phys. B 394 (1993) 73 [hep-th/9205089] [INSPIRE].

[7] J.G. Russo, L. Susskind and L. Thorlacius, Black hole evaporation in $(1+1)$-dimensions, Phys. Lett. B 292 (1992) 13 [hep-th/9201074] [INSPIRE].

[8] J.G. Russo, L. Susskind and L. Thorlacius, The Endpoint of Hawking radiation, Phys. Rev. D 46 (1992) 3444 [hep-th/9206070] [INSPIRE].

[9] J.G. Russo, L. Susskind and L. Thorlacius, Cosmic censorship in two-dimensional gravity, Phys. Rev. D 47 (1993) 533 [hep-th/9209012] [InSPIRE].

[10] J. Brown, Lower Dimensional Gravity, World Scientific Publishing Co. Pte. Ltd., Singapore (1988) DOI.

[11] L. Thorlacius, Black hole evolution, Nucl. Phys. B Proc. Suppl. 41 (1995) 245 [hep-th/9411020] [INSPIRE].

[12] D. Grumiller, W. Kummer and D.V. Vassilevich, Dilaton gravity in two-dimensions, Phys. Rept. 369 (2002) 327 [hep-th/0204253] [INSPIRE].

[13] J. Ambjørn, J. Jurkiewicz and R. Loll, The Spectral Dimension of the Universe is Scale Dependent, Phys. Rev. Lett. 95 (2005) 171301 [hep-th/0505113] [InSPIRE].

[14] P. Hořava, Spectral Dimension of the Universe in Quantum Gravity at a Lifshitz Point, Phys. Rev. Lett. 102 (2009) 161301 [arXiv:0902.3657] [inSPIRE].

[15] J.R. Mureika and D. Stojkovic, Detecting Vanishing Dimensions Via Primordial Gravitational Wave Astronomy, Phys. Rev. Lett. 106 (2011) 101101 [arXiv:1102.3434] [INSPIRE].

[16] L. Anchordoqui, D.C. Dai, M. Fairbairn, G. Landsberg and D. Stojkovic, Vanishing Dimensions and Planar Events at the LHC, Mod. Phys. Lett. A 27 (2012) 1250021 [arXiv: 1003.5914] [INSPIRE].

[17] D. Stojkovic, Vanishing dimensions: A review, Mod. Phys. Lett. A 28 (2013) 1330034 [arXiv: 1406.2696] [INSPIRE].

[18] R. Loll, Quantum Gravity from Causal Dynamical Triangulations: A Review, Class. Quant. Grav. 37 (2020) 013002 [arXiv: 1905.08669] [INSPIRE].

[19] S. Carlip, Dimension and Dimensional Reduction in Quantum Gravity, Class. Quant. Grav. 34 (2017) 193001 [arXiv:1705.05417] [INSPIRE].

[20] S. Sachdev and J. Ye, Gapless spin fluid ground state in a random, quantum Heisenberg magnet, Phys. Rev. Lett. 70 (1993) 3339 [cond-mat/9212030] [INSPIRE].

[21] A. Kitaev, A simple model of quantum holography (part 1), talk at KITP, April 7, 2015, http://online.kitp.ucsb.edu/online/entangled15/kitaev/.

[22] A. Kitaev, A simple model of quantum holography (part 2), talk at KITP, May 27, 2015, http://online.kitp.ucsb.edu/online/entangled15/kitaev2/. 
[23] A. Almheiri and J. Polchinski, Models of AdS $S_{2}$ backreaction and holography, JHEP 11 (2015) 014 [arXiv: 1402.6334] [INSPIRE].

[24] J. Maldacena, D. Stanford and Z. Yang, Conformal symmetry and its breaking in two dimensional Nearly Anti-de-Sitter space, PTEP 2016 (2016) 12C104 [arXiv:1606.01857] [INSPIRE].

[25] J. Maldacena and D. Stanford, Remarks on the Sachdev-Ye-Kitaev model, Phys. Rev. D 94 (2016) 106002 [arXiv: 1604.07818] [INSPIRE].

[26] K. Jensen, Chaos in AdS 2 Holography, Phys. Rev. Lett. 117 (2016) 111601 [arXiv: 1605.06098] [INSPIRE].

[27] V. Rosenhaus, An introduction to the SYK model, J. Phys. A 52 (2019) 323001 [arXiv: 1807.03334] [INSPIRE].

[28] G. Sárosi, AdS 2 holography and the SYK model, PoS Modave2017 (2018) 001 [arXiv: 1711.08482] [INSPIRE].

[29] D.A. Trunin, Pedagogical introduction to the Sachdev-Ye-Kitaev model and two-dimensional dilaton gravity, Usp. Fiz. Nauk 191 (2021) 225 [arXiv:2002.12187] [InSPIRE].

[30] R. Jackiw, Lower Dimensional Gravity, Nucl. Phys. B 252 (1985) 343 [InSPIRE].

[31] C. Teitelboim, Gravitation and Hamiltonian Structure in Two Space-Time Dimensions, Phys. Lett. B 126 (1983) 41 [InSPIRE].

[32] R.B. Mann, S.M. Morsink, A.E. Sikkema and T.G. Steele, Semiclassical gravity in (1+1)-dimensions, Phys. Rev. D 43 (1991) 3948 [InSPIRE].

[33] A. Vilenkin and E.P.S. Shellard, Cosmic Strings and Other Topological Defects, Cambridge University Press, U.K. (2000).

[34] T. Vachaspati, Kinks And Domain Walls, Cambridge University Press, U.K. (2006).

[35] V. Dzhunushaliev, V. Folomeev and M. Minamitsuji, Thick brane solutions, Rept. Prog. Phys. 73 (2010) 066901 [arXiv: 0904.1775] [INSPIRE].

[36] Y.-X. Liu, Introduction to Extra Dimensions and Thick Braneworlds, (2018), DOI [arXiv: 1707.08541] [INSPIRE].

[37] H.-S. Shin and K.-S. Soh, Black hole formation by sine-Gordon solitons in two-dimensional dilaton gravity, Phys. Rev. D 52 (1995) 981 [hep-th/9501045] [INSPIRE].

[38] H.M. Johng, H.S. Shin and K.S. Soh, sine-Gordon solitons coupled with dilaton gravity in two-dimensional space-time, Phys. Rev. D 53 (1996) 801 [INSPIRE].

[39] J. Gegenberg and G. Kunstatter, The Geometrodynamics of sine-Gordon solitons, Phys. Rev. D 58 (1998) 124010 [hep-th/9807042] [INSPIRE].

[40] M. Cadoni, 2-D extremal black holes as solitons, Phys. Rev. D 58 (1998) 104001 [hep-th/9803257] [INSPIRE].

[41] C. Vaz and L. Witten, Soliton induced singularities in 2-D gravity and their evaporation, Class. Quant. Grav. 12 (1995) 2607 [gr-qc/9504037] [INSPIRE].

[42] J. Yan and X.M. Qiu, Sinh-Gordon matter field and a solvable model in two-dimensional gravity, Gen. Rel. Grav. 30 (1998) 1319 [INSPIRE].

[43] J. Yan, S.-J. Wang and B.-Y. Tao, A solvable model in two-dimensional gravity coupled to a nonlinear matter field, Commun. Theor. Phys. 35 (2001) 19 [INSPIRE].

[44] B. Stoetzel, Two-dimensional gravitation and Sine-Gordon solitons, Phys. Rev. D 52 (1995) 2192 [gr-qc/9501033] [INSPIRE]. 
[45] K. Skenderis and P.K. Townsend, Gravitational stability and renormalization group flow, Phys. Lett. B 468 (1999) 46 [hep-th/9909070] [INSPIRE].

[46] O. DeWolfe, D.Z. Freedman, S.S. Gubser and A. Karch, Modeling the fifth-dimension with scalars and gravity, Phys. Rev. D 62 (2000) 046008 [hep-th/9909134] [InSPIRE].

[47] M. Gremm, Four-dimensional gravity on a thick domain wall, Phys. Lett. B 478 (2000) 434 [hep-th/9912060] [INSPIRE].

[48] A. Alonso Izquierdo, W. García Fuertes and J. Mateos Guilarte, Self-gravitating kinks in two-dimensional pseudo-Riemannian universes, Phys. Rev. D 101 (2020) 036020 [arXiv: 1911.08167] [INSPIRE].

[49] L. Randall and R. Sundrum, An Alternative to compactification, Phys. Rev. Lett. 83 (1999) 4690 [hep-th/9906064] [INSPIRE].

[50] L. Randall and R. Sundrum, A Large mass hierarchy from a small extra dimension, Phys. Rev. Lett. 83 (1999) 3370 [hep-ph/9905221] [INSPIRE].

[51] M. Eto and N. Sakai, Solvable models of domain walls in $N=1$ supergravity, Phys. Rev. $D$ 68 (2003) 125001 [hep-th/0307276] [INSPIRE].

[52] Y.-i. Takamizu and K.-i. Maeda, Collision of domain walls in asymptotically anti de Sitter spacetime, Phys. Rev. D 73 (2006) 103508 [hep-th/0603076] [InSPIRE].

[53] D. Bazeia, R. Menezes and R. da Rocha, A Note on Asymmetric Thick Branes, Adv. High Energy Phys. 2014 (2014) 276729 [arXiv: 1312.3864] [INSPIRE].

[54] J. Omotani, P.M. Saffin and J. Louko, Colliding branes and big crunches, Phys. Rev. D 84 (2011) 063526 [arXiv:1107.3938] [INSPIRE].

[55] M. Giovannini, Gauge invariant fluctuations of scalar branes, Phys. Rev. D 64 (2001) 064023 [hep-th/0106041] [INSPIRE].

[56] M. Giovannini, Localization of metric fluctuations on scalar branes, Phys. Rev. D 65 (2002) 064008 [hep-th/0106131] [INSPIRE].

[57] M. Giovannini, Scalar normal modes of higher dimensional gravitating kinks, Class. Quant. Grav. 20 (2003) 1063 [gr-qc/0207116] [INSPIRE].

[58] Y. Zhong and Y.-X. Liu, Linearization of thick K-branes, Phys. Rev. D 88 (2013) 024017 [arXiv: 1212.1871] [INSPIRE].

[59] V.F. Mukhanov, H.A. Feldman and R.H. Brandenberger, Theory of cosmological perturbations, Phys. Rept. 215 (1992) 203 [INSPIRE].

[60] H. Kodama and M. Sasaki, Cosmological perturbation theory, Progr. Theoret. Phys. Suppl. 78 (1984) 1.

[61] F. Cooper, A. Khare and U. Sukhatme, Supersymmetry and quantum mechanics, Phys. Rept. 251 (1995) 267 [hep-th/9405029] [INSPIRE].

[62] N. Ikeda and K.I. Izawa, General form of dilaton gravity and nonlinear gauge theory, Prog. Theor. Phys. 90 (1993) 237 [hep-th/9304012] [INSPIRE].

[63] K. Takahashi and T. Kobayashi, Generalized 2D dilaton gravity and kinetic gravity braiding, Class. Quant. Grav. 36 (2019) 095003 [arXiv:1812.08847] [INSPIRE].

[64] M. Bianchi, D.Z. Freedman and K. Skenderis, How to go with an RG flow, JHEP 08 (2001) 041 [hep-th/0105276] [INSPIRE].

[65] E. Kiritsis, F. Nitti and L. Silva Pimenta, Exotic RG Flows from Holography, Fortsch. Phys. 65 (2017) 1600120 [arXiv:1611.05493] [InSPIRE]. 